\title{
Theoretical and Experimental Studies of Energy Consumption in Rotor Fertilizer Spreaders Operation
}

\author{
Brovchenko Alexey Dmitrievich \\ The Department of Agricultural Science \\ Voronezh State Agrarian University \\ Voronezh, Russian Federation \\ broaldot@yandex.ru \\ Kolesnikov Nikolai Petrovich \\ The Department of Agricultural Science \\ Voronezh State Agrarian University \\ Voronezh, Russian Federation \\ nikolay2060@yandex.ru
}

\author{
Dyachkov Anatoliy Petrovich \\ The Department of Agricultural Science \\ Voronezh State Agrarian University \\ Voronezh, Russian Federation \\ Voronezh, Russian Federation \\ Sledchenko Vitaliy Anatolievich \\ The Department of Agricultural Science \\ Voronezh State Agrarian University \\ Voronezh, Russian Federation \\ sled-ko@yandex.ru
}

\begin{abstract}
Here, theoretical studies of energy consumption in rotor fertilizer spreaders operation have been given. Multi-blade rotor fertilizer spreaders have been suggested to enhance quality of fertilizer distribution. Expressions for determining all the elements of total power on the rotor shaft, such as power for translating kinetic energy to the distributed material; power for striking and granulating distributed material with blades; power for overcoming friction forces of the material on the rotor blades; power for overcoming air friction to rotor rotation; power for overcoming friction in rotor support have been determined during theoretical studies. Experimental data provide dependencies of rotor power intake on their design parameters.
\end{abstract}

Key words-fertilizer spreaders; rotor devices; energy consumption.

\section{INTRODUCTION}

Nowadays, rotor devices are used in various machines and equipment designs (trenching machines, root-extracting machines, with-covering and snow cleaning machines, tree harvesting machines and others). In agricultural sector rotor devices are used in tillage machines [1], grain throwers and fertilizer spreaders [2, 3]. In fertilizer distribution rotor spreaders overcome body fertilizer spreaders several times. It allows distributing greater amounts of solid manure in required agricultural terms.

But these spreaders have one significant drawback - high non-uniformity of material distribution which does not meet the agricultural requirements. The reason for faulty operation of rotor swath collectors and distributors is shortcomings of rotor fertilizer spreaders.
Many works have been devoted to theoretical studies of rotor devices operation, their rational design and operational parameters [4-10].

But, one of the key indicators of rotor devices operation, besides qualitative parameters of operation and productivity, is their drive energy consumption. There are not so many studies of energy consumption by rotor devices drive depending on their design and regime parameters. In most cases, simple mathematical tools are used to study energy consumption on working device drive without changes in their parameters. There are no studies of energy consumption by multi-blade rotors. While to choose rational parameters for these working devices a precise model is required to calculate energy consumption on their drive, that allows determining energy consumption for fertilizer distribution at the design stage and choose the most rational design.

Here, we describe a new design of a fertilizer spreader with rotor multi-blade working devices (Russian Federation patent № 2222883), as well as theoretical and experimental studies of its operational energy consumption.

\section{MATERIALS AND STUDIES}

To provide qualitative operational parameters during material distribution a semi-integral fertilizer spreader with multi-blade rotors from swaths has been designed (pic. 1)

During spreader motion the swath goes between the tractor wheels or caterpillars and comes to multi-blade rotors, which provide layer-by-layer fertilizer removal from the swath and distributing it for different distances from the spreader longitudal axis due to different length of blades. 


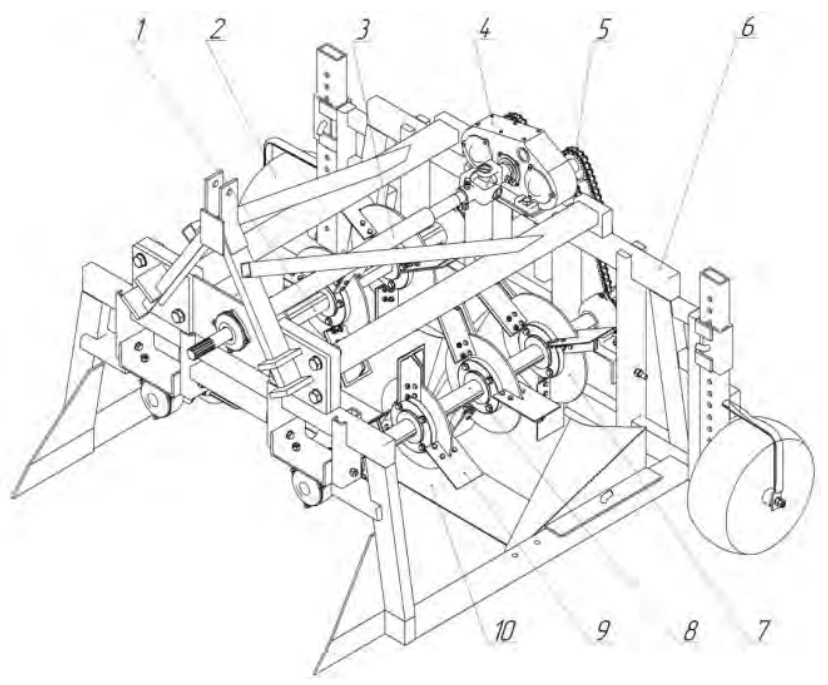

Fig. 1. Fertilizer spreader from swaths: 1,8-multiblade rotors; 2 - bearing adjusting wheeels; 3 - transmission shaft; 4 - reduction gear box; 5 -chain gears; 6 - frame; 7, 9 - rotor blades rows; 10 - lifting knife with a flow divider.

During spreader operation the total power on the rotor shaft $\mathrm{N}$ will be considered a sum of capacities:

- Power for translating kinetic energy to the distributed material $\mathrm{N}_{1}, \mathrm{KW}$;

- Power for strokes and granulating the material with blades $\mathrm{N}_{2}, \mathrm{KW}$;

- Power for overcoming material friction on rotor blades $\mathrm{N}_{3}, \mathrm{KW}$;

- Power for overcoming air friction to rotor rotation $\mathrm{N}_{4}$, $\mathrm{KW}$;

- Power for overcoming rotor supports friction $\mathrm{N}_{5}, \mathrm{KW}$.

$$
\mathrm{N}=\mathrm{N}_{1}+\mathrm{N}_{2}+\mathrm{N}_{3}+\mathrm{N}_{4}+\mathrm{N}_{5}
$$

It is necessary to take into account that multi-blade rotors have several rows of blades which take portions of fertilizers from a swath. That is why such power balance components as power for translating kinematic energy, power for overcoming friction forces of the material on blades, power for strokes and granulating fertilizers with rotor blades, and power for overcoming air friction to rotor rotation should be calculated on each blades row. But here such difficulties emerge as to calculate parameters of fertilizer particles descending from rotor blades, the calculations are unmanageable and difficult, and often are impossible without special calculating programs.

Power for translating kinematic energy to particles during their descending from the blades of $\mathrm{z}^{\text {th }}$ row is determined by the expression

$$
\mathrm{N}_{1 \mathrm{z}}=\frac{\mathrm{V}_{\mathrm{p}} \cdot \mathrm{S}_{\mathrm{z}} \cdot \gamma \cdot \mathrm{V}_{\mathrm{acpz}}^{2}}{2 \cdot \mathrm{g} \cdot 102 \cdot \eta_{\text {пр }}}=\frac{\mathrm{W}_{\mathrm{z}} \cdot \mathrm{V}_{\mathrm{acpz}}^{2}}{204 \cdot \mathrm{g} \cdot \eta_{\text {пр }}}
$$

where $V_{p}$ is forward speed of the rotor spreader, $\mathrm{m} / \mathrm{s}$;

$\mathrm{S}_{\mathrm{z}}$ is the shaft cross section area, in the capture zone of the blades of $\mathrm{z}^{\text {th }}$ row, $\mathrm{m}^{2}$;

$\gamma$ is density of distributed fertilizers, $\mathrm{kg} / \mathrm{m}^{3}$;

$\mathrm{V}_{\mathrm{acpz}}$ is an average speed of fertilizer particles descending from blades of $\mathrm{z}^{\text {th }}$ row, $\mathrm{m} / \mathrm{s}$;

$\mathrm{H}_{\text {rotor shaft }}$ is efficiency of the rotor working device shaft;

$\mathrm{W}_{\mathrm{z}}$ is rotor efficiency of $\mathrm{z}^{\text {th }}$ rotor row, $\mathrm{kg} / \mathrm{s}$.

Value of $\eta_{\text {eff }}$ can be calculated by formula

$$
\eta_{\text {eff }}=\eta_{\text {chgear }}^{2} \cdot \eta_{\text {chgear }} \cdot \eta_{\text {bearings }}
$$

where $\eta_{3}$ is cylindrical gearing efficiency(in the reduction gear);

$\eta_{\mathrm{ch}}$ is chain gear efficiency;

$\eta_{\text {bearings }}$ is roller bearing efficiency.

Total power for translating kinematic energy to particles descending from blades of all rotor rows is determined by the expression

$$
\mathrm{N}_{1}=\sum_{1}^{\mathrm{z}} \mathrm{N}_{1 \mathrm{z}}
$$

where $\mathrm{z}$ is blades rows amount, which take part in fertilizer spreading from a swath.

Here it should be taken into account that accourding to expressions (2) and (4) during operation blades of all rotor rows take portions of fertilizers from the swath simultaneously. But, accourding to the suggested design, blades of every consequent row, from the rotor beginning, are evenly off-centered to some angle counter-clockwise, forming multiple-thread coiling the rotor shafts. Besides, fertilizer portion descending from a blade of any rotor row is done by a certain time period, when the blade rotates for some angle called casting sector or blade dumping angle [11-15]. Taking into account these comments we can write the formula (4) as

$$
N_{1}=\left(\sum_{1}^{z} N_{1 z}\right) \cdot k_{\text {blades }}
$$

where kblades is a coefficient which takes into account uniform blade location of all rotor rows in a circumferential direction as well as their dumping angles.

Power spent on strokes and granulating the material with blades of the $n^{\text {th }}$ row 


$$
N_{2 n}=\frac{W_{z} \cdot V_{\text {bladesavengez }}^{2}}{204 \cdot g \cdot \eta_{\text {rotorshaft }}}
$$

where $\mathrm{V}_{\text {blades average } \mathrm{z}}$ is average linear blades travel speed of the $\mathrm{z}^{\text {th }}$ row, $\mathrm{m} / \mathrm{s}$.

$$
V_{\text {bladesavengez }}=\frac{\pi \cdot n \cdot\left(R_{H z}+R_{0 z}\right)}{30 \cdot 2}=\frac{\omega \cdot\left(R_{z}+L_{0 z}\right)}{2}
$$

where $\mathrm{n}$ is a rotor rotation speed, $\mathrm{s}^{-1}$;

$\mathrm{R}_{\mathrm{H} \mathrm{z}}$ is maximal fertilizer distance from the rotation center on the blades of the $\mathrm{z}^{\text {th }}$ row, m;

$\mathrm{R}_{0 \mathrm{z}}$ is minimal fertilizer distance from the rotation center on the blades of $z^{\text {th }}$ row, $m$.

Taking into account the uniform blade location in a circumferential direction we can say that not all the blades will be in a swath at a given time but some of them. In this regard, power determined by the expression (6), in fact will be less then estimated value. As lowering this power component depends on rotor blades location (as well as in the previous case) then it can be estimated with the coefficient $\mathrm{k}_{\text {blades. }}$ Then, the total power for strokes and material granulating will be:

$$
N_{2}=\left(\sum_{1}^{z} N_{2 z}\right) \cdot k_{\text {blades }}
$$

Power necessary to overcome material friction on radially positioned blade of $\mathrm{z}^{\text {th }}$ rotor row is expressed:

$$
N_{3 z}=\frac{f \cdot V_{\text {raveragez }}}{1000 \cdot \eta_{\text {rotorshaft }}} \cdot\left[\begin{array}{l}
\frac{4 \cdot W_{z} \cdot\left(R_{z}-L_{0 z}\right) \cdot \omega \cdot m_{z}}{g_{\pi z}}+m_{z} \cdot g \cdot \sin \left(\beta_{\text {averagez }}\right) \\
\left.+{ }^{\prime}\right)
\end{array}\right],(9)
$$

where $\mathrm{f}$ is a fertilizer friction coefficient on blades;

$\mathrm{V}_{\mathrm{r} \text { average } \mathrm{z}}$ is an average relative particles speed on the blade of $\mathrm{z}^{\text {th }}$ row, m/s; $\mathrm{kg}$;

$\mathrm{G}_{\text {onblades } \mathrm{z}}$ is an average material mass on blades of $\mathrm{z}^{\text {th }}$ row,

$\mathrm{m}_{\mathrm{z}}$ is material mass on the blades of $\mathrm{z}^{\text {th }}$ row, $\mathrm{kg}$;

$\beta_{\text {average } z}$ is a mean value of the angle between weight direction and the blade plane of the $\mathrm{z}^{\text {th }}$ row during particles descending, radius.

$$
\text { Here } \quad V_{\text {raveragez }}=\frac{V_{r z}}{2}(10)
$$

where $\mathrm{V}_{\mathrm{r} z}$ is average relative material particles speed at the end of the blade $\mathrm{z}^{\mathrm{th}}$ row, $\mathrm{m} / \mathrm{s}$.
When the material amount per 1 linear meter of the rotor blade of $z^{\text {th }}$ row

$$
g_{0 z}=\frac{W_{z}}{V_{\text {raveragez }}}=\frac{2 \cdot W_{z}}{V_{r z}}
$$

$$
g_{\text {bladesz }}=g_{0 z} \cdot\left(R_{H z}-R_{0 z}\right)=\frac{2 \cdot W_{z} \cdot\left(R_{H z}-R_{0 z}\right)}{V_{r z}}
$$

$$
\mathrm{m}_{\mathrm{z}}=\mathrm{V}_{\mathrm{p}} \cdot \frac{\varepsilon_{\mathrm{z}}}{\omega} \cdot \mathrm{S}_{\mathrm{z}} \cdot \gamma
$$

where $\varepsilon_{\mathrm{z}}$ is an angle between the sections connecting the rotation axis and the point of intersection of the extreme blade edge of $z^{\text {th }}$ row with the swath, radius.

If the blades of the rotor rows are dislocated relative to the radial position, then formula (9) receives a component of the centrifugal force in square brackets

$$
N_{3 z}=\frac{f \cdot V_{\text {raveragez }}}{1000 \cdot \eta_{\text {rotorshaft }}} \cdot\left[\begin{array}{l}
\frac{4 \cdot W_{z} \cdot\left(R_{z}-L_{0 z}\right) \cdot \omega \cdot m_{z}}{g_{\text {bladesz }}}+ \\
+m_{z} \cdot g \cdot \sin \left(\beta_{\text {averagez }}\right) \pm \\
\pm \frac{m_{z} \cdot\left(R_{z}-L_{0 z}\right) \cdot \omega^{2} \cdot \sin \left(\psi_{\text {averagez }}\right)}{2}
\end{array}\right]
$$

where $\psi_{\text {average } \mathrm{z}}$ is a mean angle value, between the centrifugal force direction and the blade plane of the $z^{\text {th }}$ row at the moment of fertilizer particles descending from the blade, radius.

When the blades are set at the angle $(-\theta)$ to the rotor radius (a negative angle - contrary the direction of rotor rotation), the $\mathrm{n}$ it is necessary to put minus before the last expression in square brackets of the equation (14). If the location angle $\theta$ is positive (the blades are inclined toward the direction of rotor rotation) then we should put plus before the expression.

As the result the total power necessary to overcome material friction forces on blades of all rotor rows, with the account of the uniform blades location over the rotor circumference can be determined by the expression

$$
N_{3}=\left(\sum_{1}^{z} N_{3 z}\right) \cdot k_{\text {blades }}
$$


During rotor rotation there are two motion reactions: reaction to the rotor blades displacement and reaction to air friction on their lateral surfaces. Power consumption for the second type of reaction in calculations is less than $1 \mathrm{KW}$, so they can be neglected.

Power spent on overcoming air friction to blades rotation of the $\mathrm{z}^{\text {th }}$ rotor row

$$
N_{4 z}=\frac{C \cdot S_{\text {totalz }} \cdot V_{\max z}^{3}}{102 \cdot 4 \cdot \eta_{\text {rotorshaft }}}
$$

where $S_{\text {total } z}$ is the total area of all working blades surfaces of $\mathrm{z}^{\mathrm{th}}$ rotor row, $\mathrm{m}^{2}$;

$\mathrm{C}$ is air mass density, $\frac{\mathrm{kg} \cdot \mathrm{s}^{2}}{\mathrm{~m}^{4}} ;$

$\mathrm{V}_{\max \mathrm{z}}$ is blade edges linear speed of the $\mathrm{z}^{\mathrm{th}}$ rotor row, $\mathrm{m} / \mathrm{s}$;

Here

$$
C=\frac{\gamma_{\text {air }}}{2 \cdot g}
$$

where $\gamma_{\text {air }}$ is air density, $\mathrm{kg} / \mathrm{m}^{3}$.

$$
\begin{gathered}
\mathrm{V}_{\operatorname{maxz}}=\frac{\pi \cdot \mathrm{R}_{\mathrm{z}} \cdot \mathrm{n}}{30}=\mathrm{R}_{\mathrm{z}} \cdot \omega \\
S_{\text {total }}=Z \cdot S_{0 z} \\
S_{0 z}=\left(R_{z}-O A\right) \cdot b_{\text {bladesz }}
\end{gathered}
$$

where $\mathrm{S}_{0 \mathrm{z}}$ is one blade area of the $\mathrm{z}^{\text {th }}$ row, $\mathrm{m}^{2}$;

$\mathrm{Z}$ is blades amount in the row;

$\mathrm{B}_{\text {blades } \mathrm{z}}$ is blades width of the $\mathrm{z}^{\text {th }}$ row, $\mathrm{m}$.

Total power spent on overcoming air friction force to all rotor blades rotation

$$
\mathrm{N}_{4}=\left(\sum_{1}^{\mathrm{z}} \mathrm{N}_{4 \mathrm{z}}\right)
$$

Power spent on overcoming friction resistance in rotor supports can be determined by the formula

$$
N_{5}=\frac{f_{1} \cdot(G+q) \cdot D \cdot \omega}{102 \cdot \eta_{\text {rotorshaft }}}
$$

where $f_{1}$ is a friction coefficient in rotor supports;

$\mathrm{G}$ is rotor mass, $\mathrm{kg}$;

$\mathrm{q}$ is material mass on rotor blades, $\mathrm{kg}$;

$\mathrm{D}$ is rotor supports diameter, $\mathrm{m}$

$$
q=\left(\sum_{1}^{z} m_{z}\right) \cdot k_{\text {blades }}
$$

By expression (1) we can determine total power consumption for rotor operation. Total power consumption for the shaft of two rotors will be equal

$$
N_{\text {rotorshaft }}=2 \cdot N
$$

To determine the influence of multi-blade rotors design parameters on power consumption experiments were conducted when with the measuring instruments the rotor driving torque was recorded and its rotation frequency was determined. According to the received data we calculated the power spent on Multiblade rotor shaft depending on the blades width at different feed per second of manure and radial rotor blades position. Picture 2 shows the dependence graph of power consumption on multi-blade rotor shaft on blades width.

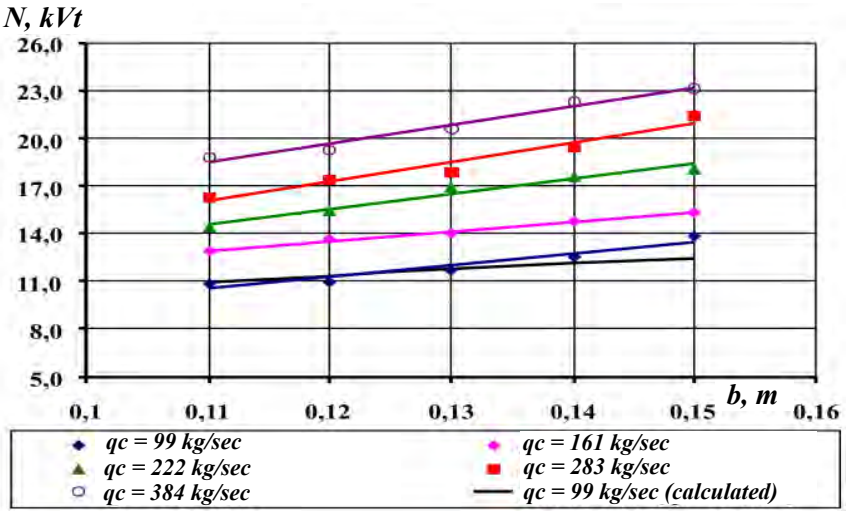

Fig. 2. Dependence of power on rotor shaft on blades width

As the graphs show the blades width influences insignificantly the rotor power consumption. As the given parameter increases the power increases at all material feeding per second. It is caused by power consumption increase for overcoming air resistance to rotor rotation. Whereas the least power consumption are at b blades $=110 \mathrm{~mm}$, it is necessary to use blades with width $b_{\text {blades }}=140 \mathrm{~mm}$, as they provide the necessary quality of fertilizer distribution. That is why at radial blade positioning the consumed rotor power will be $\mathrm{N}=$ $12,5 \ldots 22,3 \mathrm{KW}$.

Basing on the data found we have determined the coefficient accounting uniform blades positioning of all rotor rows along the circumference of rotation, as well as their 
discharge angles (expressions (5), (8), (15), (21)). The coefficient numerical value was $\mathrm{k}_{\text {blades }}=0,59 \ldots 0,87$. Where the least coefficient value corresponds to utilization of tworow rotors, the greater to the four-row rotors for certain fertilizer feeding per second. To compare theoretical and experimental data picture 2 shows the calculated dependence received by equations (1)-(24) for fertilizer feeding $\mathrm{q}_{\mathrm{s}}=99$ $\mathrm{kg} / \mathrm{s}$. As we can see these dependences correspond each other well.

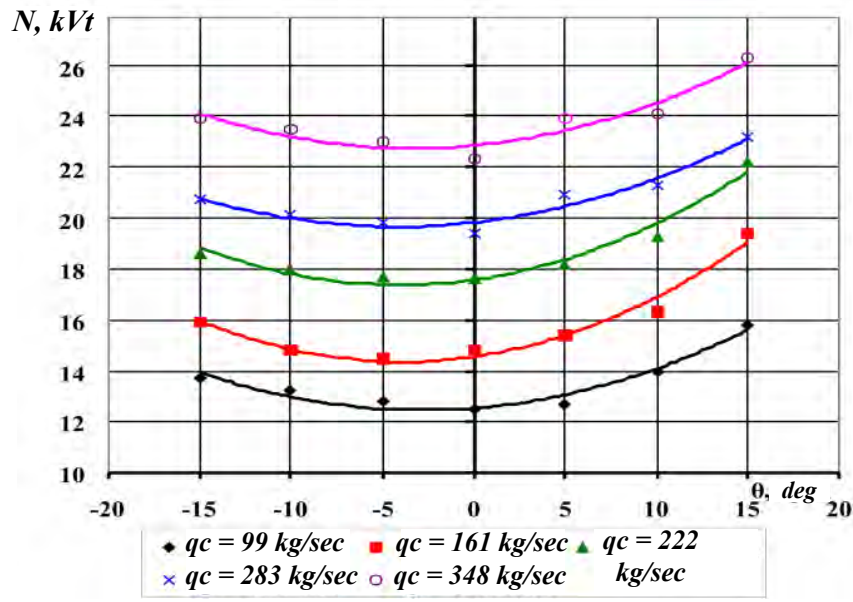

Fig. 3. Dependence of power on rotors shaft on bladesw angle inclination

Figure 3 shows dependence graphs of rotor power consumed on inclination angle of rotor rows blades.

The graph analysis shows that when blades inclination angles are $\theta=-15^{\circ} \ldots+5^{\circ}$ the power consumed is nearly the same at all fertilizer feedings per second. When the blade inclination angle is $\theta=+5^{\circ} \ldots+15^{\circ}$ the consumed power on rotor shafts increases. It can be explained by the following. At the given angles of blades inclination the average relative fertilizer particles speed decreases, and the power to translate them kinematic energy decreases (this has been proved by theoretical studies). But here power consumption to overcome friction forces of fertilizer particles on blades increases. Besides, time of material position on the blades increases and angles of dissipation sector increase increases that results in rotor resistance torque increase.

Thus the least power consumption is at angles of rotor blades inclination $\theta=-10^{\circ} \ldots 0^{\circ}$ and are equal to $\mathrm{N}=$ $12,5 \ldots 22,3 \mathrm{KW}$ at the given range of feeding per second

\section{CONCLUSION}

The received expressions for determining power consumption in multi-blade rotor working devices allow taking into account varying parameters of the swath, rotor spreading working devices design and kinematic parameters, as well as grounding rational parameters of rotor spreading working devices during theoretical studies from the point of view of energy consumption.

\section{References}

[1] Md. A. Matin, J. M. Fielke, and J. M. A. Desbiolles, "Furrow parameters in rotary strip-tillage: Effect of blade geometry and rotary speed,' Biosystems Engineering, vol. 118, pp. 7-15, February 2014.

[2] A. Przywara, "The Impact of Structural and Operational Parameters of the Centrifugal Disc spreader on the Spatial Distribution of Fertilizer,' Agriculture and Agricultural Science Procedia, vol. 7, pp. 215-222, 2015 .

[3] J. Koko, and T. Virin, "Optimization of a fertilizer spreading process," Mathematics and Computers in Simulation, vol. 79, 10, pp. 3099-3109, June 2009.

[4] A. Aphale, N. Bolander, J. Park, L. Shaw, J. Svec, and C, Wassgren, "Granular Fertiliser Particle Dynamics on and off a Spinner Spreader,' Biosystems Engineering, vol. 85, issue 3, pp. 319-329, July 2003

[5] J. W. Hofstee, "Handling and Spreading of Fertilizers: Part 5, The Spinning Disc Type Fertilizer Spreader," Journal of Agricultural Engineering Research, vol. 62, issue 3, pp. 143-162, November 1995.

[6] S. Villette, F. Cointault, E. Piron, and B. Chopinet, "Centrifugal Spreading: an Analytical Model for the Motion of Fertiliser Particles on a Spinning Disc," Biosystems Engineering, vol. 92, issue 2, pp. 157-164, October 2005

[7] P. Van Liedekerke, E. Tijskens, and H. Ramon, "Discrete element simulations of the influence of fertiliser physical properties on the spread pattern from spinning disc spreaders," Biosystems Engineering, 102, issue 4, pp. 392-405, April 2009.

[8] A. Aan, and M. Heinloo, "Motion of a granule on fertilizer spreading disc," International Symposium on Agricultural Engineering. Opatija, CROATIA vol. 42, pp. 101-112, 2014.

[9] E. -M. Abbou-ou-cherif, E. Piron, A. Chateauneuf, D. Miclet, R. Lenain, and J. Koko, "On-the-field simulation of fertilizer spreading: Part 1 - Modeling," Computers and Electronics in Agriculture, vol. 142 part A, pp. 235-247, November 2017.

[10] A. D. Brovchenko, A. P. D'achkov, N. P. Kolesnikov, and V. A Sledchenko, "Mathematical simulation of rotor tool technological proces," IOP: Earth and Environmental Science (EES), in press.

[11] L.Yang, L. Chen, J. Zhang, H. Liu, Z. Sun, H. Sun, and L. Zheng, "Fertilizer sowing simulation of a variable-rate fertilizer applicator based on EDEM," IFAC-PapersOnLine, vol. 51, issue 17, pp.418-423, 2018.

[12] S. Yinyan, C. Man, W.Xiaochan, M. O. Odhiambo, and D. Weimin, "Numerical simulation of spreading performance and distribution pattern of centrifugal variable-rate fertilizer applicator based on DEM software," Computers and Electronics in Agriculture, vol. 144, pp. 249 259, January 2018.

[13] S. Yinyan, H. Zhichao, W. Xiaochan, M. O. Odhiambo, and D. Weimin, "Motion analysis and system response of fertilizer feed apparatus for paddy Variable-Rate fertilizer spreader," Computers and Electronics in Agriculture, vol. 153, pp. 239-247, October 2018.

[14] P.Van Liedekerke, E. Tijskens, E. Dintwa, F. Rioual, J. Vangeyte, and H. Ramona, "DEM simulations of the particle flow on a centrifugal fertilizer spreader,"Powder Technology, vol. 190, issue 3, pp. 348-360, 25 March 2009.

[15] E. Dieudé-Fauvel, P. Héritier, J.C. Roux, "Impact of sludge mechanica behaviour on spatial distribution parameters obtained with centrifugal spreader: Preliminary study," Engineering in Agriculture, Environment and Food, vol. 9, issue 3, pp. 242-249, July 2016 